\title{
Nucleon transversity generalized form factors with twisted mass fermions
}

\section{Alexandrou}

Department of Physics, University of Cyprus, P.O. Box 20537, 1678 Nicosia, Cyprus, and Computation-based Science and Technology Research Center, Cyprus Institute, 20 Kavafi Str., Nicosia 2121, Cyprus

E-mail: alexand@ucy.ac.cy

\section{Constantinou*}

Department of Physics, University of Cyprus, P.O. Box 20537, 1678 Nicosia, Cyprus

E-mail: marthac@ucy.ac.cy

\section{K. Jansen}

NIC, DESY, Platanenallee 6, D-15738 Zeuthen, Germany

E-mail: karl.jansen@desy.de

\section{G. Koutsou}

Computation-based Science and Technology Research Center, Cyprus Institute, 20 Kavafi Str., Nicosia 2121, Cyprus

E-mail: g.koutsou@cyi.ac.cy

\section{H. Panagopoulos}

Department of Physics, University of Cyprus, P.O. Box 20537, 1678 Nicosia, Cyprus E-mail: hariseucy.ac.cy

\begin{abstract}
We present results on the nucleon tensor form factors and first moment of the transversity distribution using maximally twisted mass fermions. We analyze two $N_{f}=2+1+1$ ensembles having pion masses of $213 \mathrm{MeV}$ and $373 \mathrm{MeV}$ with lattice spacing $a=0.064 \mathrm{fm}$ and $a=0.082 \mathrm{fm}$, respectively. First results using an $N_{f}=2$ ensemble of twisted mass fermions with a clover term at a physical pion mass and lattice spacing $a=0.094 \mathrm{fm}$ are also presented. The renormalization function for the local tensor form factors is evaluated non-perturbatively with a perturbative subtraction of $\mathscr{O}\left(a^{2}\right)$-terms, while for the first moment of the transversity we use a perturbative estimate. Results are given in the $\overline{\mathrm{MS}}$ scheme at a scale of $\mu=2 \mathrm{GeV}$, and are compared with recent results obtained using different discretization schemes.
\end{abstract}

31st International Symposium on Lattice Field Theory - LATTICE 2013

July 29 - August 3, 2013

Mainz, Germany

\footnotetext{
*Speaker.
} 


\section{Introduction}

Lattice QCD calculations of observables related to the structure of baryons are now being carried out using simulations of the theory with pion mass close or even at the physical value $[1,2$, $3,4,5]$. Nucleon observables that are under intense experimental study are the Generalized Parton Distributions (GPDs), which encode important information on nucleon structure. The GPDs can be accessed in high energy processes where QCD factorization applies, and the amplitude can be written in terms of the convolution of a hard perturbative kernel with the GPDs. The twist-2 GPDs, which are studied in this paper, are defined by the matrix element:

$$
F_{\Gamma}\left(x, \xi, q^{2}\right)=\frac{1}{2} \int \frac{d \lambda}{2 \pi} e^{i x \lambda}\left\langle p^{\prime}\left|\bar{\psi}(-\lambda n / 2) \Gamma \mathscr{P} e^{i g \int_{-\lambda / 2}^{\lambda / 2} d \alpha n \cdot A(n \alpha)} \psi(\lambda n / 2)\right| p\right\rangle,
$$

where $\left|p^{\prime}\right\rangle$ and $|p\rangle$ are one-particle states, $q=p^{\prime}-p, \xi=-n \cdot q / 2, x$ is the momentum fraction, and $n$ is a light-like vector collinear to $P=\left(p+p^{\prime}\right) / 2$ and such that $P \cdot n=1$. The gauge link $\mathscr{P} \exp (\ldots)$ is necessary for gauge invariance. In model calculations it is often set to one, which amounts to working with QCD in the light-like gauge $A \cdot n=0$, but on the lattice such a gauge fixing is not necessary. In the forward limit, for which $\xi=0$ and $q^{2}=0$, GPDs reduce to the ordinary parton distributions, namely the longitudinal momentum, $q(x)$, the helicity, $\Delta q(x)$, and transversity, $\delta q(x)$, distributions; in this paper we restrict to the transverity which represents the net number of quarks with transverse polarization in a transversely polarized nucleon. The first few Mellin moments of the transversity parton distribution are of particular interest

$$
\left\langle x^{n}\right\rangle_{\delta q}=\int_{0}^{1} d x x^{n}\left[\delta q(x)+(-1)^{n+1} \delta \bar{q}(x)\right], \quad \delta q=q_{\top}+q_{\perp} .
$$

The matrix elements of the light-cone operator as defined in Eq. (1.1) cannot be extracted from correlators in euclidean lattice QCD but an operator product expansion can be carried out leading to

$$
\mathscr{O}_{\top}^{\mu v \mu_{1} \ldots \mu_{n-1}}=\bar{q} \sigma^{[\mu\{v]} i D^{\mu_{1}} \ldots i D^{\left.\mu_{n-1}\right\}} q .
$$

The curly brackets represent a symmetrization over indices and subtraction of traces, while the square brackets represent antisymmetrization over indices. Here we study the cases $n=0,1$, which amount to calculating the local and one-derivative tensor currents, respectively. The matrix elements of these operators are parameterized in terms of the generalized form factors (GFFs) $A_{T 10}, B_{T 10}, \widetilde{A}_{T 10}$ and $A_{T 20}, B_{T 20}, \widetilde{A}_{T 20}, \widetilde{B}_{T 21}$ depending only on $q^{2}=\left(p^{\prime}-p\right)^{2}$ :

$$
\begin{aligned}
\left\langle\left\langle\bar{q}(0) i \sigma^{\mu v} q(0)\right\rangle\right\rangle & =\left\langle\left\langle i \sigma^{\mu v}\right\rangle\right\rangle A_{T 10}\left(q^{2}\right)+\left\langle\left\langle\frac{\gamma^{[\mu} \Delta^{v]}}{2 m_{N}}\right\rangle\right\rangle B_{T 10}\left(q^{2}\right)+\left\langle\left\langle\frac{\bar{P}^{[\mu} \Delta^{v]}}{m_{N}^{2}}\right\rangle \widetilde{A}_{T 10}\left(q^{2}\right),\right. \\
\left\langle\left\langle\bar{q}(0) \mathscr{O}_{T}^{\mu v \mu_{1}}(0) q(0)\right\rangle\right\rangle & =\mathscr{A}_{\mu \nu} \mathscr{S}_{v \mu_{1}}\left\{\left\langle i \sigma^{\mu v} \bar{P}^{\mu_{1}}\right\rangle A_{T 20}\left(q^{2}\right)+\left\langle\left\langle\frac{\gamma^{[\mu} \Delta^{v]}}{2 m_{N}} \bar{P}^{\mu_{1}}\right\rangle B_{T 20}\left(q^{2}\right)\right.\right. \\
& \left.+\left\langle\frac{\bar{P}^{[\mu} \Delta^{v]}}{m_{N}^{2}} \bar{P}^{\mu_{1}}\right\rangle \widetilde{A}_{T 20}\left(q^{2}\right)+\left\langle\left\langle\frac{\gamma^{[\mu} \bar{P}^{v]}}{m_{N}} \Delta^{\mu_{1}}\right\rangle\right\rangle \widetilde{B}_{T 21}\left(q^{2}\right)\right\} .
\end{aligned}
$$

In the forward limit we can directly obtain $A_{T 10}(0)=\langle 1\rangle_{\delta q(x)}$ and $A_{T 20}(0)=\langle x\rangle_{\delta q(x)}$. 


\section{Evaluation on the lattice}

In the present work we employ the twisted mass fermion (TMF) action with $N_{f}=2+1+1$ dynamical quarks [20] and the Iwasaki improved gauge action. We also present results for an ensemble of $N_{f}=2$ TMFs with a clover term and tree-level Symanzik gauge action. Using standard techniques, the GFFs are extracted from dimensionless ratios of correlation functions, involving two-point and three-point functions:

$$
\begin{aligned}
G\left(\vec{q}, t_{f}-t_{i}\right) & =\sum_{\vec{x}_{f}} e^{-i\left(\vec{x}_{f}-\vec{x}_{i}\right) \cdot \vec{q}} \Gamma_{\beta \alpha}^{0}\left\langle J_{\alpha}\left(t_{f}, \vec{x}_{f}\right) \bar{J}_{\beta}\left(t_{i}, \vec{x}_{i}\right)\right\rangle, \\
G^{\mu v \mu_{1}}\left(\Gamma^{k}, \vec{q}, t\right) & =\sum_{\vec{x}, \vec{x}_{f}} e^{i\left(\vec{x}-\vec{x}_{i}\right) \cdot \vec{q}} \Gamma_{\beta \alpha}^{k}\left\langle J_{\alpha}\left(t_{f}, \vec{x}_{f}\right) \mathscr{O}^{\mu v \mu_{1}}(t, \vec{x}) \bar{J}_{\beta}\left(t_{i}, \vec{x}_{i}\right)\right\rangle .
\end{aligned}
$$

We consider kinematics for which the final momentum $\vec{p}^{\prime}=0$ and we employed the fixed-sink method which requires a fixed time separation between the sink and the source, $t_{f}-t_{i}$. The projection matrices $\Gamma^{0}$ and $\Gamma^{k}$ are given by

$$
\Gamma^{0}=\frac{1}{4}\left(\mathbb{1}+\gamma_{0}\right), \quad \sum_{k=1}^{3} \Gamma^{k}=\Gamma^{0} i \gamma_{5} \sum_{k=1}^{3} \gamma_{k}
$$

We use the standard proton interpolating field with Gaussian smeared quark fields to increase the overlap with the proton state and decrease overlap with excited states. We also apply APE-smearing to the gauge fields $U_{\mu}$ [5]. For matrix elements of isovector operators the disconnected contributions are zero up to lattice artifacts. For the isoscalar local tensor we have computed the disconnected diagram, which was found to be very small [7]. We form an appropriate ratio of three- and two- functions

$$
R^{\mu v}\left(\Gamma^{k}, \vec{q}, t\right)=\frac{G^{\mu v}\left(\Gamma^{k}, \vec{q}, t\right)}{G\left(\overrightarrow{0}, t_{f}-t_{i}\right)} \times \sqrt{\frac{G\left(\vec{p}, t_{f}-t\right) G\left(\overrightarrow{0}, t-t_{i}\right) G\left(\overrightarrow{0}, t_{f}-t_{i}\right)}{G\left(\overrightarrow{0}, t_{f}-t\right) G\left(\vec{p}, t-t_{i}\right) G\left(\vec{p}, t_{f}-t_{i}\right)}},
$$

which is optimized because it does not contain potentially noisy two-point functions at large separations and because correlations between its different factors reduce the statistical noise. For sufficiently large time separations of the source and the sink, this ratio becomes time-independent:

$$
\lim _{t_{f}-t \rightarrow \infty t-t_{i} \rightarrow \infty} \lim ^{\mu v}\left(\Gamma^{\lambda}, \vec{q}, t\right)=\Pi^{\mu v}\left(\Gamma^{\lambda}, \vec{q}\right) .
$$

From the plateau values of the renormalized asymptotic ratio $\Pi\left(\Gamma^{k}, \vec{q}\right)_{R}=Z \Pi\left(\Gamma^{k} j, \vec{q}\right)$ the nucleon matrix elements of the operators can be extracted. All values of $\vec{q}$ corresponding to the same $q^{2}$, the two choices of projector matrices $\Gamma^{0}$ and $\sum_{k} \Gamma^{k}$ and the relevant orientations $\mu, v, \rho$ of the operators lead to an over-constrained system of equations, which is solved in the least-squares sense via a singular value decomposition of the coefficient matrix. All quantities will be given in Euclidean space with $Q^{2} \equiv-q^{2}$ being the Euclidean momentum transfer squared. Both projectors $\Gamma^{0}$ and $\sum_{k} \Gamma^{k}$ are required to obtain all GFFs at non-zero momentum. Not all combinations of the indices $\mu, v, \mu_{1}$, are nessecary but we use all possibilities in order to increase statistics. In Fig. 1 we show representative plateau for the ratios of the local tensor and the one derivative tensor operators at $\beta=1.95$, for different $\vec{Q}$-components. 


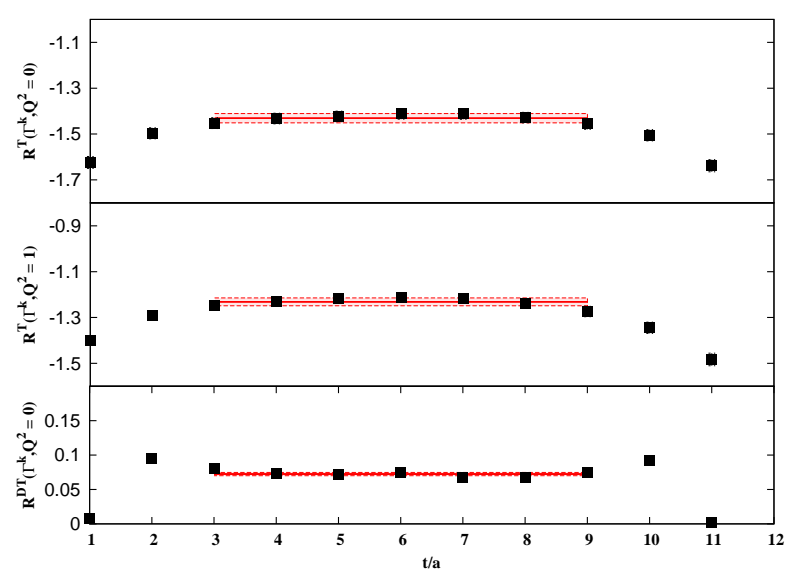

Figure 1: $R^{\mu v}$ (upper two) and $R^{\mu v \mu_{1}}$ for representative choices of the momentum. The solid lines with the bands indicate the fitted ranges and plateau values with their jackknife errors.
In this study we use sequential propagators already produced for the computation of other nucleon matrix elements with $t_{f}-$ $t_{i} \sim 1 \mathrm{fm}$ namely, for the $N_{f}=2+1+1 \mathrm{TMF}$ ensembles we use $\left(t_{f}-t_{i}\right) / a=12$ for $\beta=$ 1.95, $\left(t_{f}-t_{i}\right) / a=18$ for $\beta=2.10$ and for the $N_{f}=2$ TMF with a clover term ensemble, $\left(t_{f}-t_{i}\right) / a=12,14$. For the latter ensemble we find that the results are compatible within error bars with the data for $\left(t_{f}-t_{i}\right) / a=14$ carrying larger statistical errors. Thus, in the plots we only show the results for $\left(t_{f}-t_{i}\right) / a=12$.

\section{Renormalization}

We determine the necessary renormalization functions for the local tensor operator non perturbatively in the RI' scheme by employing a momentum source at the vertex [8], which leads to high statistical accuracy and the evaluation of the vertex for any operator at no significant additional computational cost. For the details of the non-perturbative renormalization see Ref. [9]. In the RI' scheme the renormalization functions are determined in the chiral limit. For the renormalization of our $N_{f}=2+1+1$ ensembles, ETMC has generated $N_{f}=4$ ensembles at the same $\beta$ values, so that the chiral limit can be taken. To improve our final estimates obtained from the continuum extrapolation we have also computed the Green's functions related to the renormalization functions in perturbation theory up to $\mathscr{O}\left(a^{2}\right)$ terms $[10,11]$; we perform a perturbative subtraction of these $\mathscr{O}\left(a^{2}\right)$-terms. This subtracts the leading cut-off effects yielding, in general, a weak dependence of the renormalization functions on $(a p)^{2}$ and the $(a p)^{2} \rightarrow 0$ limit can be reliably taken; this can be seen in Fig. 2 for the two $N_{f}=2+1+1$ ensembles. As an example, we present the perturbative terms that we subtract for the Iwasaki gluonic action and clover coefficient $c_{\mathrm{sw}}=0$ :

$$
a^{2} \frac{g^{2} C_{F}}{16 \pi^{2}}\left[0.2341 \mu^{2}+\frac{8}{3} \frac{\mu 4}{\mu^{2}}+\log \left(a^{2} \mu^{2}\right)\left(\frac{7271}{60000} \mu^{2}-\frac{28891}{30000} \frac{\mu 4}{\mu^{2}}\right)\right], \quad\left(\mu 4 \equiv \sum_{i=1,4} \mu_{i}^{4}\right) .
$$

For the renormalization functions of the one-derivative tensor operator, $Z_{\mathrm{DT}}$, we use our perturbative results [9], which we compute for general action parameters. For Iwasaki gluons the expression for $Z_{\mathrm{DT}}$ in the $\mathrm{RI}^{\prime}$ scheme is:

$$
Z_{D T}(p=\bar{\mu})=1+\frac{g^{2} C_{F}}{16 \pi^{2}}\left(2.3285-2.2795 c_{\mathrm{sw}}-1.0117 c_{\mathrm{sw}}^{2}-3 \log \left(a^{2} \bar{\mu}^{2}\right)\right) .
$$

The renormalization functions are converted to the $\overline{\mathrm{MS}}$ scheme at a scale of $\mu=2 \mathrm{GeV}$ using the conversion factors of Refs. [12,13]. For the non-perturbative estimate of $Z_{T}$ we first subtract 
the $\mathscr{O}\left(a^{2}\right)$ perturbative terms and then apply the conversion to the $\overline{\mathrm{MS}}$ scheme. The values of $Z_{T}^{\overline{\mathrm{MS}}}(2 \mathrm{GeV})$ which we use in this paper are given below, where the numbers in the parenthesis correspond to the statistical error. As mentioned earlier, we use our perturbative results on $Z_{D T}$ to renormalize the traversity moment:

$$
\begin{array}{rr}
\beta=1.95, N_{f}=2+1+1: Z_{T}=0.625(2), & Z_{D T}=1.019 \\
\beta=2.10, N_{f}=2+1+1: Z_{T}=0.664(1), & Z_{D T}=1.048 \\
\beta=2.10, N_{f}=2, c_{s w}=1.58: Z_{T}=0.914(1), & Z_{D T}=1.104
\end{array}
$$
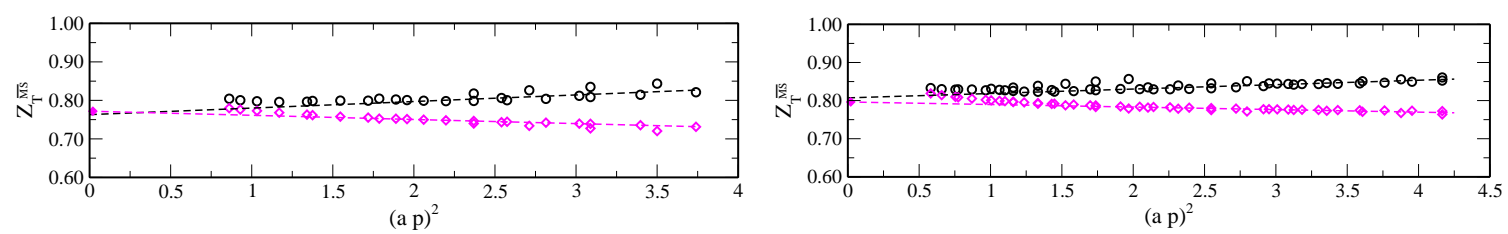

Figure 2: $Z_{T}^{\overline{\mathrm{MS}}}(2 \mathrm{GeV})$ for $N_{f}=4$ at $\beta=1.95$ (left) and $\beta=2.10$ (right). Black circles are the unsubtracted data and the magenta diamonds the data after subtracting the perturbative $\mathscr{O}\left(a^{2}\right)$-terms. The solid diamond at $(a p)^{2}=0$ is the value obtained after performing a linear extrapolation on the subtracted data.

\section{Lattice Results}

In this section we present results for the isovector and isoscalar nucleon tensor charge $g_{T} \equiv$ $A_{T 10}(0)$, the first moment of the transversity $\langle x\rangle_{\delta q} \equiv A_{T 20}(0)$, and compare with results using other lattice discretizations. The renormalization functions for the isoscalar quantities receive a contribution from a disconnected diagram. For the Wilson gluonic action, the correction was computed perturbatively and found to be very small [14]. We assume that the correction is also small for the gauge action used here and it is therefore neglected.

In Fig. 3 we collect our results for the tensor charge. These are computed at different lattice spacings ranging from $a \sim 0.1 \mathrm{fm}$ to $a \sim 0.06 \mathrm{fm}$, and at different volumes. As can be seen, there are no sizable cut-off effects. A comparison with other lattice discretizations $[15,16,17,18]$ shows that all lattice results are in good agreement.

In Fig. 4 we show as an example the $Q^{2}$ dependence of $A_{T 10}^{u-d}$ for the twisted mass results at various pion masses (left panel) and a comparison with results from $N_{f}=2$ clover fermions [19] (right panel). The latter correspond to a range of values for $m_{\pi} \sim 600-1000 \mathrm{MeV}$. Despite the difference in the pion masses, the results are in good agreement.

From the matrix elements of the one-derivative tensor operator we extract $A_{T 20}$, which is the GFF that can be computed directly from the lattice data in the forward limit. In Fig. 5 we collect our data for the isovector case (left panel) and we compare with results from $N_{f}=2$ clover fermions [19] (right panel). Opposed to $A_{T 10}$, we find that $A_{T 20}$ is not the same at different values of the pion mass. This could be due to the perturbative renormalization and/or a pion mass dependence.

\section{Conclusions}

The tensor charge is evaluated for a range of pion masses including the physical value. Our values are in agreement with the values obtained using clover and domain wall fermions. Neglect- 

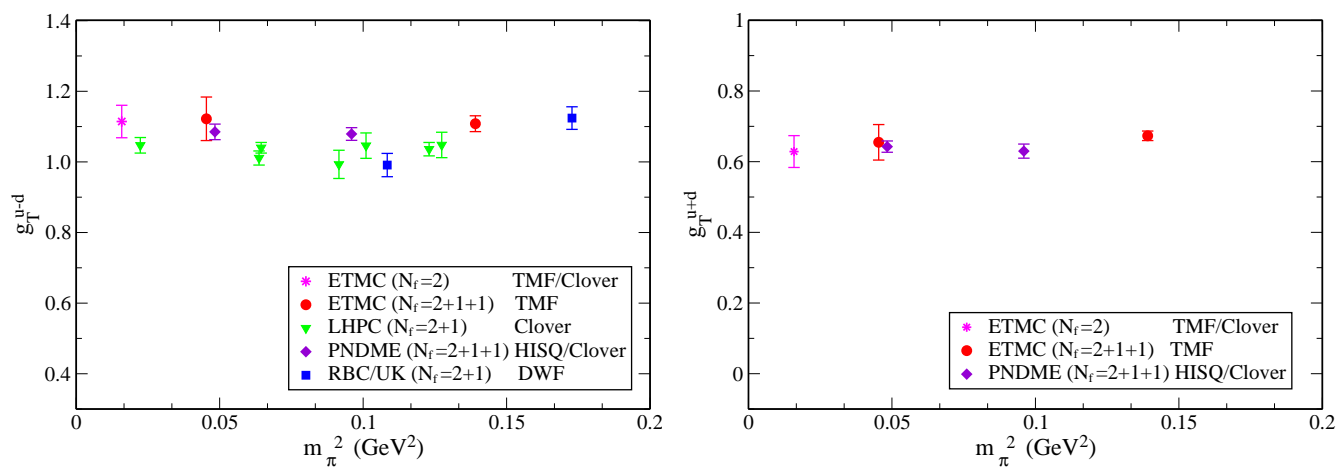

Figure 3: The nucleon isovector (left panel) and isoscalar (right panel) tensor charge for $N_{f}=2$ TMF with a clover term (magenta asterisk) and $N_{f}=2+1+1$ TMF (red circles), as well as results using other lattice actions: green triangles correspond to $N_{f}=2+1$ clover fermions [17], violet diamonds to $N_{f}=2+1+1$ clover on HISQ fermions [18], blue squares to $N_{f}=2+1$ domain wall fermions [15] and $N_{f}=2$ clover fermions [16].
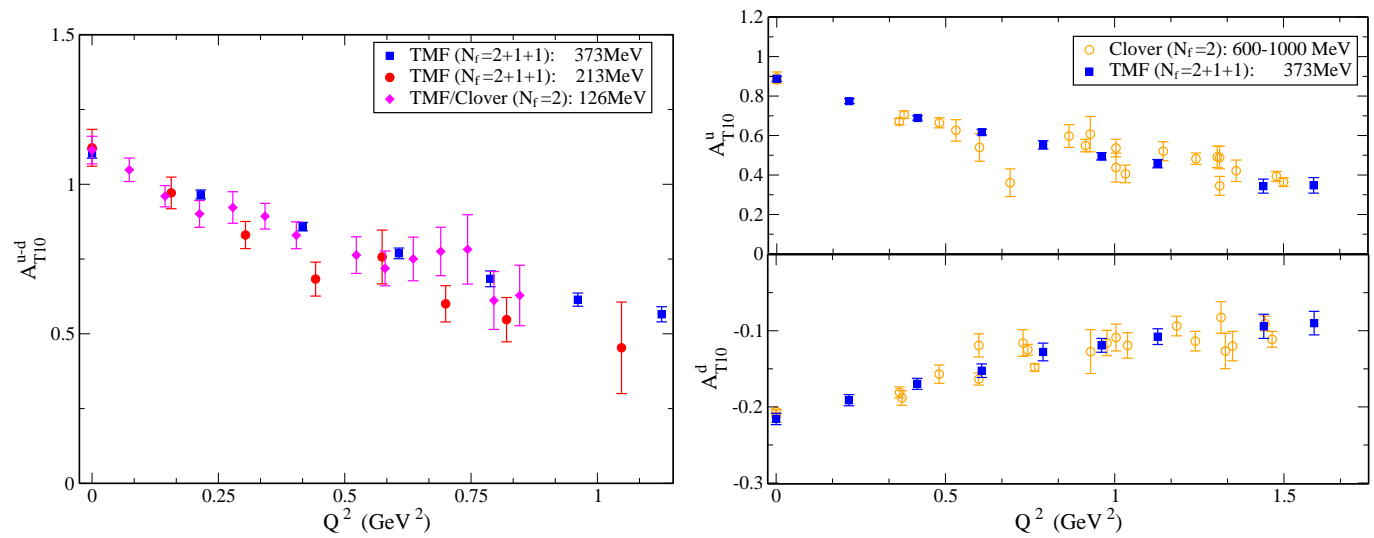

Figure 4: Left panel: The dependence of $A_{T 10}^{u-d}$ on the momentum transfer, $Q^{2}$, for i) $N_{f}=2$ TMF with a clover term at $m_{\pi}=126 \mathrm{MeV}$ (magenta diamonds), ii) $N_{f}=2+1+1 \mathrm{TMF}$ at $m_{\pi}=213 \mathrm{MeV}$ (red circles) and at $m_{\pi}=373 \mathrm{MeV}$ (blue squares). Right panel: a comparison between $N_{f}=2+1+1 \mathrm{TMF}$ at $m_{\pi}=373$ $\mathrm{MeV}$ (blue squares) and $N_{f}=2$ clover fermions at $m_{\pi} \sim 600-1000 \mathrm{MeV}$ (orange circles) [16].

ing disconnected contributions we find at the physical point $g_{T}^{u}=0.87(4)$ and $g_{T}^{d}=0.25(3)$. The first moment of the transversity distribution is also computed for the first time in the chiral regime, albeit with a perturbative renormalization. The next step will be to compute the non-perturbative renormalization for the transversity distribution.

Acknowledgments: M. C. would like to thank the Cyprus Research Promotion Foundation

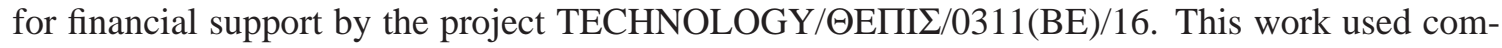
putational resources provided by PRACE, JSC, Germany.

\section{References}

[1] P. Hagler et al. [LHPC], Phys. Rev. D 77 (2008) 094502 [arXiv:0705.4295].

[2] S. N. Syritsyn et al., Phys. Rev. D 81 (2010) 034507 [arXiv:0907.4194]. 

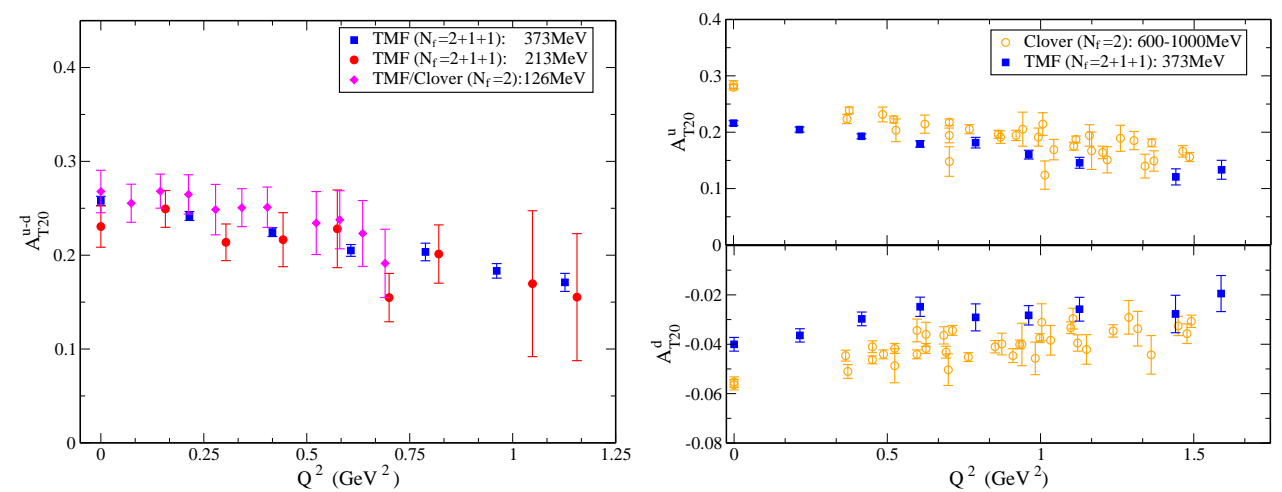

Figure 5: $A_{T 20}$ versus $Q^{2}$. The notation is the same as that of Fig. 4.

[3] D. Brommel et al. [QCDSF/UKQCD], PoS LAT 2007 (2007) 158 [arXiv:0710.1534].

[4] T. Yamazaki, Y. Aoki, T. Blum, H. -W. Lin, S. Ohta, S. Sasaki, R. Tweedie and J. Zanotti, Phys. Rev. D 79 (2009) 114505 [arXiv:0904.2039].

[5] C. Alexandrou, M. Constantinou, S. Dinter, V. Drach, K. Jansen, C. Kallidonis and G. Koutsou, Phys. Rev. D 88 (2013) 014509 [arXiv:1303.5979].

[6] R. Baron et al. [ETMC], Comput. Phys. Commun. 182 (2011) 299 [arXiv:1005.2042].

[7] A. Abdel-Rehim, C. Alexandrou, M. Constantinou, V. Drach, K. Hadjiyiannakou, K. Jansen, G. Koutsou and A. Vaquero, arXiv:1310.6339.

[8] M. Göckeler, R. Horsley, H. Oelrich, H. Perlt, D. Petters, P. E. L. Rakow, A. Schafer and G. Schierholz et al., Nucl. Phys. B 544 (1999) 699 [hep-lat/9807044].

[9] C. Alexandrou, M. Constantinou, T. Korzec, H. Panagopoulos and F. Stylianou, Phys. Rev. D 83 (2011) 014503 [arXiv:1006.1920].

[10] M. Constantinou, V. Lubicz, H. Panagopoulos and F. Stylianou, JHEP 0910 (2009) 064 [arXiv:0907.0381].

[11] C. Alexandrou, M. Constantinou, T. Korzec, H. Panagopoulos and F. Stylianou, Phys. Rev. D 86 (2012) 014505 [arXiv:1201.5025].

[12] J. A. Gracey, Nucl. Phys. B 662 (2003) 247 [hep-ph/0304113].

[13] J. A. Gracey, JHEP 0610 (2006) 040 [hep-ph/0609231].

[14] A. Skouroupathis and H. Panagopoulos, Phys. Rev. D 79 (2009) 094508 [arXiv:0811.4264].

[15] Y. Aoki, T. Blum, H. -W. Lin, S. Ohta, S. Sasaki, R. Tweedie, J. Zanotti and T. Yamazaki, Phys. Rev. D 82 (2010) 014501 [arXiv:1003.3387].

[16] D. Pleiter et al. [QCDSF/UKQCD], PoS LATTICE 2010 (2010) 153 [arXiv:1101.2326].

[17] J. R. Green, J. W. Negele, A. V. Pochinsky, S. N. Syritsyn, M. Engelhardt and S. Krieg, Phys. Rev. D 86 (2012) 114509 [arXiv:1206.4527].

[18] T. Bhattacharya, S. D. Cohen, R. Gupta, A. Joseph and H. -W. Lin, arXiv:1306.5435.

[19] M. Göckeler et al. [QCDSF/UKQCD], Phys. Lett. B 627 (2005) 113 [hep-lat/0507001].

[20] R. Baron et al. [ETMC], Comput. Phys. Commun. 182 (2011) 299 [arXiv:1005.2042]. 